\title{
Polynomial Sequences Associated with the Moments of Hypergeometric Weights
}

\section{Diego DOMINICI}

Department of Mathematics, State University of New York at New Paltz,

1 Hawk Dr., New Paltz, NY 12561-2443, USA

E-mail: dominicd@newpaltz.edu

URL: https://www2.newpaltz.edu/ dominicd/

Received November 23, 2015, in final form April 25, 2016; Published online April 29, 2016

http://dx.doi.org/10.3842/SIGMA.2016.044

\begin{abstract}
We present some families of polynomials related to the moments of weight functions of hypergeometric type. We also consider different types of generating functions, and give several examples.
\end{abstract}

Key words: moments; hypergeometric functions; generating functions; Stieltjes transform

2010 Mathematics Subject Classification: 44A60; 33C20; 05A15

Dedicated to my daughter Malena, luz de mi vida!

\section{Introduction}

Let $\left\{\mu_{n}\right\}$ be a sequence of complex numbers and $\mathcal{L}: \mathbb{C}[x] \rightarrow \mathbb{C}$ be a linear functional defined by

$$
\mathcal{L}\left(x^{n}\right)=\mu_{n}, \quad n=0,1, \ldots
$$

Then, $\mathcal{L}$ is called the moment functional determined by the formal moment sequence $\left\{\mu_{n}\right\}$. The number $\mu_{n}$ is called the moment of order $n$. The task of finding an explicit representation for the functional $\mathcal{L}$ is called a moment problem [1, 24, 32].

A sequence $\left\{\Pi_{n}(x)\right\} \subset \mathbb{C}[x]$, with $\operatorname{deg}\left(\Pi_{n}\right)=n$ is called an orthogonal polynomial sequence with respect to $\mathcal{L}$ provided that [4]

$$
\mathcal{L}\left(\Pi_{n} \Pi_{m}\right)=K_{n} \delta_{n, m}, \quad n, m=0,1, \ldots,
$$

where $K_{n} \neq 0$ and $\delta_{n, m}$ is Kronecker's delta.

The moments play a fundamental role in the theory of orthogonal polynomials since, among other results, we have the determinantal representation

$$
\Pi_{n}(x)=C_{n}\left|\begin{array}{cccc}
\mu_{0} & \mu_{1} & \cdots & \mu_{n} \\
\mu_{1} & \mu_{2} & \cdots & \mu_{n+1} \\
\vdots & \vdots & \ddots & \vdots \\
\mu_{n-1} & \mu_{n} & \cdots & \mu_{2 n-1} \\
1 & x & \cdots & x^{n}
\end{array}\right|,
$$

for some normalization constant $C_{n} \neq 0$, with

$$
\left|\begin{array}{cccc}
\mu_{0} & \mu_{1} & \cdots & \mu_{n} \\
\mu_{1} & \mu_{2} & \cdots & \mu_{n+1} \\
\vdots & \vdots & \ddots & \vdots \\
\mu_{n} & \mu_{n+1} & \cdots & \mu_{2 n}
\end{array}\right| \neq 0, \quad n=0,1, \ldots
$$

\footnotetext{
${ }^{\star}$ This paper is a contribution to the Special Issue on Orthogonal Polynomials, Special Functions and Applications. The full collection is available at http://www.emis.de/journals/SIGMA/OPSFA2015.html
} 
Given their importance, it is very striking that they are not explicitly listed in the standard books on orthogonal polynomials, or even in encyclopedic texts such as [23]. In fact, the only place where we found a comprehensive enumeration of the moments of classical orthogonal polynomials was the recent article [28], based on the results obtained in the Ph.D. Thesis of the first author $[27]^{1}$.

In this paper, we focus our attention on linear functionals defined by

$$
\mathcal{L}(f)=\sum_{x=0}^{\infty} f(x) \rho(x ; \vec{\alpha}, \vec{\beta}, c),
$$

where the weight function $\rho(x ; \vec{\alpha}, \vec{\beta}, c)$ is of the form

$$
\rho(x ; \vec{\alpha}, \vec{\beta}, c)=\frac{(\vec{\alpha})_{x}}{(\vec{\beta}+1)_{x}} \frac{c^{x}}{x !}
$$

with

$$
(\vec{\alpha})_{x}=\left(\alpha_{1}\right)_{x}\left(\alpha_{2}\right)_{x} \cdots\left(\alpha_{p}\right)_{x}, \quad(\vec{\beta}+1)_{x}=\left(\beta_{1}+1\right)_{x}\left(\beta_{2}+1\right)_{x} \cdots\left(\beta_{q}+1\right)_{x},
$$

and $(a)_{x}$ denotes the Pochhammer symbol (also called shifted or rising factorial) defined by [29, $(5.2 .4)]$

$$
(a)_{0}=1, \quad(a)_{x}=a(a+1) \cdots(a+x-1), \quad x \in \mathbb{N},
$$

or by

$$
(a)_{x}=\frac{\Gamma(a+x)}{\Gamma(a)}, \quad a+x \neq 0,-1, \ldots,
$$

and $\Gamma(z)$ is the Gamma function. Unless stated otherwise, we always assume that

$$
\beta_{i}>-1, \quad 1 \leq i \leq q,
$$

and we will use the notation

$$
\vec{\alpha}=\alpha_{1}, \alpha_{1}, \ldots, \alpha_{p}, \quad \vec{\beta}+1=\beta_{1}+1, \beta_{2}+1, \ldots, \beta_{q}+1 .
$$

Note that we have

$$
\frac{\rho(x ; \vec{\alpha}, \vec{\beta}, c)}{\rho(x ; \vec{\alpha}, \vec{\beta}, c)}=\frac{\eta(x)}{\phi(x+1)},
$$

with

$$
\phi(x)=x\left(x+\beta_{1}\right)\left(x+\beta_{2}\right) \cdots\left(x+\beta_{q}\right), \quad \eta(x)=c\left(x+\alpha_{1}\right)\left(x+\alpha_{2}\right) \cdots\left(x+\alpha_{p}\right) .
$$

Hence, the weight function $\rho(x ; \vec{\alpha}, \vec{\beta}, c)$ satisfies the Pearson equation (see [30] or [31, (6.3)])

$$
\Delta(\phi \rho)=(\eta-\phi) \rho,
$$

where

$$
\Delta f(x)=f(x+1)-f(x)
$$

\footnotetext{
${ }^{1}$ We thank one of the referees for stressing this fact.
} 
is the forward difference operator. If we define $s$ by

$$
s=\max \{\operatorname{deg}(\phi)-2, \operatorname{deg}(\phi-\eta)-1\},
$$

the sequence of polynomials orthogonal with respect to $\mathcal{L}$ is called semiclassical of class $s$ [18, 25].

Weight functions of the form (2) are also related to discrete probability distributions (Poisson, Pascal, binomial, hypergeometric, etc.) $[21]^{2}$. The Generalized Hypergeometric probability distributions were studied by Adrienne W. Kemp in her Ph.D. Thesis [22] $]^{3}$. An excellent reference outlining the connections between the theory of probability and orthogonal polynomials is [31].

In [5], it was pointed out that since

$$
\mu_{n}(c)=\sum_{x=0}^{\infty} x^{n} \rho(x ; \vec{\alpha}, \vec{\beta}, c),
$$

one has

$$
\mu_{n+1}(c)=\sum_{x=0}^{\infty} x^{n} x \rho(x ; \vec{\alpha}, \vec{\beta}, c)=\vartheta \mu_{n}(c),
$$

where the differential operator $\vartheta$ is defined by [29, (16.8.2)]

$$
\vartheta f(c)=c \frac{d f}{d c} .
$$

Successive applications of (5) give

$$
\mu_{n}=\vartheta^{n} \mu_{0}
$$

and it follows that the first moment $\mu_{0}$ determines the whole sequence $\left\{\mu_{n}\right\}$. If we use the operational formula [26]

$$
\vartheta^{k}=\sum_{k=0}^{n}\left\{\begin{array}{l}
n \\
k
\end{array}\right\} c^{k} \frac{d^{k}}{d c^{k}}
$$

we have

$$
\mu_{n}(c)=\sum_{k=0}^{n}\left\{\begin{array}{l}
n \\
k
\end{array}\right\} c^{k} \frac{d^{k} \mu_{0}}{d c^{k}},
$$

where $\left\{\begin{array}{l}n \\ k\end{array}\right\}$ denote the Stirling numbers of the second kind defined by [29, (26.8)]

$$
\left\{\begin{array}{l}
n \\
k
\end{array}\right\}=\frac{1}{k !} \sum_{j=0}^{k}\left(\begin{array}{l}
k \\
j
\end{array}\right)(-1)^{k-j} j^{n} .
$$

From (2), we have

$$
\mu_{0}(c)=\sum_{x=0}^{\infty} \rho(x ; \vec{\alpha}, \vec{\beta}, c)={ }_{p} F_{q}\left[\begin{array}{l}
\vec{\alpha} \\
\vec{\beta}
\end{array} ; c\right],
$$

where ${ }_{p} F_{q}$ is the generalized hypergeometric function [29, (16.2.1)]. Depending on the values of $p$ and $q$, we have to consider three different cases:

\footnotetext{
${ }^{2}$ We thank one of the editors for suggesting this reference.

${ }^{3}$ Unfortunately, we haven't been able to obtain a copy of it.
} 
1. If $p<q+1, \mu_{0}(c)$ is an entire function of $c$.

2. If $p=q+1, \mu_{0}(c)$ is analytic inside the unit disk $|c|<1$.

3. If $p>q+1$, the series (9) diverges for $c \neq 0$, unless one or more of the top parameters $\alpha_{i}$ is a negative integer. If we take $\alpha_{1}=-N$, with $N \in \mathbb{N}$, then $\mu_{0}(c)$ becomes a polynomial of degree $N$.

Using the formula $[29,(16.3 .1)]$

$$
\frac{d^{n}}{d c^{n}}{ }_{p} F_{q}\left[\begin{array}{l}
\vec{a} \\
\vec{b}
\end{array} ; c\right]=\frac{(\vec{a})_{n}}{(\vec{b})_{n}}{ }_{p} F_{q}\left[\begin{array}{l}
\vec{a}+n \\
\vec{b}+n
\end{array} ; c\right],
$$

in (7), we have

$$
\mu_{n}(c)=\sum_{k=0}^{n}\left\{\begin{array}{l}
n \\
k
\end{array}\right\} c^{k} \frac{(\vec{\alpha})_{k}}{(\vec{\beta}+1)_{k}}{ }_{p} F_{q}\left[\begin{array}{c}
\vec{\alpha}+k \\
\vec{\beta}+1+k
\end{array} ; c\right] .
$$

Although (10) seems to give an explicit formula for the moments, this type of sums (to our knowledge) can't be evaluated in closed form.

An alternative is to consider generalized moments, defined by [2]

$$
\nu_{n}=\mathcal{L}\left(\varphi_{n}\right),
$$

where $\left\{\varphi_{n}\right\} \subset \mathbb{C}[x]$, with $\operatorname{deg}\left(\varphi_{n}\right)=n$. Choosing ${ }^{4}$

$$
\varphi_{n}(x)=(x-n+1)_{n},
$$

we get

$$
\nu_{n}(c)=\sum_{x=0}^{\infty}(x-n+1)_{n} \frac{(\vec{\alpha})_{x}}{(\vec{\beta}+1)_{x}} \frac{c^{x}}{x !}=\frac{(\vec{\alpha})_{k}}{(\vec{\beta}+1)_{k}} p F_{q}\left[\begin{array}{c}
\vec{\alpha}+k \\
\vec{\beta}+1+k
\end{array} ; c\right] .
$$

Since $[29,(26.8 .10)]$

$$
x^{n}=\sum_{k=1}^{n}\left\{\begin{array}{l}
n \\
k
\end{array}\right\}(x-k+1)_{k},
$$

we have

$$
\mu_{n}(c)=\mathcal{L}\left(x^{n}\right)=\sum_{k=1}^{n}\left\{\begin{array}{l}
n \\
k
\end{array}\right\} \mathcal{L}\left(\varphi_{k}\right)=\sum_{k=1}^{n}\left\{\begin{array}{l}
n \\
k
\end{array}\right\} \nu_{k}(c),
$$

and we recover (10).

In a series of papers $[7,8,10,13,14,16]$, we studied polynomial solutions of differentialdifference equations of the form

$$
P_{n+1}(x)=A_{n}(x) P_{n}^{\prime}(x)+B_{n}(x) P_{n}(x), \quad n \geq 0,
$$

where $P_{0}(x)=1$, and $A_{n}(x), B_{n}(x)$ are polynomials of degree at most 2 and 1 respectively. In this article, we consider some extensions of (12) to the multidimensional case, with $P_{n}(x)$ replaced by a vector $\vec{P}_{n}(x)$, and $B_{n}(x)$ replaced by a matrix $\mathbf{B}_{n}(x)$.

\footnotetext{
${ }^{4}$ As suggested by one of the anonymous referees.
} 
In [19] and [20], it was shown that some families of orthogonal polynomials can be represented as moments of probability measures. In this work, we do the opposite and express the moments $\mu_{n}(c)$ in terms of some polynomials $\vec{P}_{n}(c)$.

The paper is organized as follows: in Section 2, we derive a differential-difference equation for the polynomials $\vec{P}_{n}(c)$ associated to the moments $\mu_{n}(c)$. We also find formulas for the exponential generating functions and Stieltjes transforms of $\mu_{n}(c)$ and $\vec{P}_{n}(c)$. In Section 3 , we apply our results to most of the families of discrete semiclassical orthogonal polynomials of class 1 studied in [15], except for limiting and $c=1$ cases. Finally, in Section 4, we outline some conclusions and possible future directions.

\section{Main results}

The function $\mu_{0}(c)$ satisfies the differential equation [29, (16.8.3)]

$$
\left[\vartheta\left(\vartheta+\beta_{1}\right) \cdots\left(\vartheta+\beta_{q}\right)-c\left(\vartheta+\alpha_{1}\right) \cdots\left(\vartheta+\alpha_{p}\right)\right] \mu_{0}=0
$$

where $\vartheta$ was defined in (6). We can rewrite the ODE (13) as

$$
\begin{aligned}
& \vartheta^{q+1} \mu_{0}=\sum_{k=0}^{q} \sigma_{k}(c) \vartheta^{k} \mu_{0}, \quad q>p-1, \\
& (1-c) \vartheta^{q+1} \mu_{0}=\sum_{k=0}^{q} \sigma_{k}(c) \vartheta^{k} \mu_{0}, \quad q=p-1, \\
& c \vartheta^{p} \mu_{0}=\sum_{k=0}^{p-1} \sigma_{k}(c) \vartheta^{k} \mu_{0}, \quad q<p-1,
\end{aligned}
$$

where the coefficients $\sigma_{k}(c)$ are linear functions of $c$.

Introducing the quantities

$$
\vec{\mu}(c)=\left[\begin{array}{c}
\mu_{0}(c) \\
\mu_{1}(c) \\
\vdots \\
\mu_{\xi}(c)
\end{array}\right], \quad \xi=\max \{p-1, q\}
$$

and

$$
(\lambda, \tau)= \begin{cases}(1,0), & q+1>p \\ (1,-1), & q+1=p \\ (0,1), & q+1<p\end{cases}
$$

we can rewrite (14) as

$$
(\lambda+c \tau) \mu_{\xi+1}=\sum_{k=0}^{\xi} \sigma_{k}(c) \mu_{k} .
$$

If we define the $(\xi+1) \times(\xi+1)$ matrix $\mathbf{M}(c)$ by

$$
\mathbf{M}_{i, j}=\left\{\begin{array}{lll}
\sigma_{j}, & i=\xi+1, & 0 \leq j \leq \xi \\
\lambda+c \tau, & j=i+1, & 0 \leq i \leq \xi-1, \\
0, & \text { otherwise, }
\end{array}\right.
$$


we get

$$
\mathbf{M} \vec{\mu}=(\lambda+c \tau) \vartheta \vec{\mu} .
$$

We can now state our main result.

Proposition 1. Let the $(\xi+1)$-vector polynomials $\vec{P}_{n}(c)$ be defined by

$$
\vec{P}_{0}(c)=\left[\begin{array}{c}
1 \\
0 \\
\vdots \\
0
\end{array}\right]
$$

and

$$
\vec{P}_{n+1}=c(\lambda+c \tau) \frac{d \vec{P}_{n}}{d c}+\left(\mathbf{M}^{T}-n \tau c \mathbf{I}\right) \vec{P}_{n}, \quad n=0,1, \ldots,
$$

where $\mathbf{I}$ is the $(\xi+1) \times(\xi+1)$ identity matrix. Then,

$$
\mu_{n}(c)=(\lambda+c \tau)^{-n} \vec{P}_{n}(c) \cdot \vec{\mu}(c), \quad n=0,1, \ldots
$$

Proof. Using (15) and (17), we have

$$
\begin{aligned}
\vec{P}_{n+1} \cdot \vec{\mu} & =(\lambda+c \tau) \vartheta \vec{P}_{n} \cdot \vec{\mu}+\left(\mathbf{M}^{T}-n \tau c \mathbf{I}\right) \vec{P}_{n} \cdot \vec{\mu} \\
& =(\lambda+c \tau) \vartheta \vec{P}_{n} \cdot \vec{\mu}+\vec{P}_{n} \cdot(\mathbf{M}-n \tau c \mathbf{I}) \vec{\mu} \\
& =(\lambda+c \tau) \vartheta \vec{P}_{n} \cdot \vec{\mu}+\vec{P}_{n} \cdot(\lambda+c \tau) \vartheta \vec{\mu}-n \tau c \vec{P}_{n} \cdot \vec{\mu} .
\end{aligned}
$$

Multiplying by $(\lambda+c \tau)^{-n-1}$ we get

$$
\begin{aligned}
(\lambda+c \tau)^{-n-1} \vec{P}_{n+1} \cdot \vec{\mu} & =(\lambda+c \tau)^{-n} \vartheta \vec{P}_{n} \cdot \vec{\mu}+\vec{P}_{n} \cdot(\lambda+c \tau)^{-n} \vartheta \vec{\mu}-n \tau c(\lambda+c \tau)^{-n-1} \vec{P}_{n} \cdot \vec{\mu} \\
& =\vartheta\left[(\lambda+c \tau)^{-n} \vec{P}_{n} \cdot \vec{\mu}\right] .
\end{aligned}
$$

Thus, the sequence

$$
r_{n}=(\lambda+c \tau)^{-n} \vec{P}_{n} \cdot \vec{\mu}
$$

satisfies the recurrence $r_{n+1}=\vartheta r_{n}$ with initial condition

$$
r_{0}=\vec{P}_{0} \cdot \vec{\mu}=\mu_{0} .
$$

From (5), we conclude that $r_{n}=\mu_{n}$.

\subsection{Generating functions}

Let's consider the exponential generating function for the moments, defined by the formal power series

$$
G_{\mu}(c, w)=\sum_{n=0}^{\infty} \mu_{n}(c) \frac{w^{n}}{n !} .
$$

Given that

$$
\mu_{n+1}=c \mu_{n}^{\prime},
$$


we have [33]

$$
\frac{\partial G_{\mu}}{\partial w}=c \frac{\partial G_{\mu}}{\partial c}
$$

with general solution

$$
G_{\mu}(c, w)=H\left(c e^{w}\right)
$$

for some function $H(w)$. But since

$$
G_{\mu}(c, 0)=\mu_{0}(c),
$$

we conclude that

$$
G_{\mu}(c, w)=\mu_{0}\left(c e^{w}\right)
$$

Using (18), we see that the exponential generating function for the polynomials $\vec{P}_{n}(c)$

$$
\vec{G}_{P}(c, w)=\sum_{n=0}^{\infty} \vec{P}_{n}(c) \frac{w^{n}}{n !}
$$

satisfies

$$
G_{\mu}(c,(\lambda+\tau c) w)=\vec{G}_{P}(c, w) \cdot \vec{\mu}(c),
$$

or, using (19),

$$
\vec{G}_{P}(c, w) \cdot \vec{\mu}(c)=\mu_{0}\left(c e^{(\lambda+\tau c) w}\right) .
$$

\subsection{Stieltjes transform}

A different type of generating function for the moments that is very important in the theory of orthogonal polynomials is the Stieltjes transform (or $Z$ transform), that can be defined by the formal Laurent series

$$
S_{\mu}(c, z)=\sum_{n=0}^{\infty} \frac{\mu_{n}(c)}{z^{n+1}} .
$$

We have

$$
S_{\mu}(c, z)=\sum_{n=0}^{\infty} \frac{\mu_{n}(c)}{z^{n+1}}=\sum_{n=-1}^{\infty} \frac{\mu_{n+1}(c)}{z^{n+2}}=\frac{1}{z} \sum_{n=-1}^{\infty} \frac{\mu_{n+1}(c)}{z^{n+1}}=\frac{1}{z}\left[\mu_{0}(c)+\sum_{n=0}^{\infty} \frac{\mu_{n+1}(c)}{z^{n+1}}\right] .
$$

Hence,

$$
\sum_{n=0}^{\infty} \frac{\mu_{n+1}(c)}{z^{n+1}}=z S_{\mu}(c, z)-\mu_{0}(c)
$$

and using (5), we get

$$
c \frac{\partial S_{\mu}}{\partial c}=z S_{\mu}-\mu_{0}(c) .
$$


From (4), we have

$$
S_{\mu}(0, z)=\frac{1}{z}
$$

and solving (22) we obtain

$$
S_{\mu}(c, z)=-c^{z} \int_{0}^{c} \frac{\mu_{0}(x)}{x^{z+1}} d x=-\int_{0}^{1} \frac{\mu_{0}(c t)}{t^{z+1}} d t .
$$

Using the recurrence relation for the Gamma function, we can write

$$
-\int_{0}^{1} \frac{\mu_{0}(c t)}{t^{z+1}} d t=\frac{1}{z} \frac{\Gamma(1-z)}{\Gamma(1) \Gamma(-z)} \int_{0}^{1} \frac{\mu_{0}(c t)}{t^{z+1}} d t
$$

and therefore

$$
S_{\mu}(c, z)=\frac{1}{z}{ }_{p+1} F_{q+1}\left[\begin{array}{c}
-z, \vec{\alpha} \\
1-z, \vec{\beta}+1
\end{array} ; c\right]
$$

where we have used the integral representation [29, (16.5.2)]

$$
{ }_{p+1} F_{q+1}\left[\begin{array}{ll}
\alpha_{0}, & \vec{\alpha} \\
\beta_{0}, & \vec{\beta}
\end{array} ; c\right]=\frac{\Gamma\left(\beta_{0}\right)}{\Gamma\left(\alpha_{0}\right) \Gamma\left(\beta_{0}-\alpha_{0}\right)} \int_{0}^{1} t^{\alpha_{0}-1}(1-t)^{\beta_{0}-\alpha_{0}-1}{ }_{p} F_{q}\left[\begin{array}{l}
\vec{\alpha} \\
\vec{\beta}
\end{array} ; c t\right] d t .
$$

We derived (23) in [12] using a different approach.

If we define the Stieltjes transform of $\vec{P}_{n}(c)$ by

$$
\vec{S}_{P}(c, z)=\sum_{n=0}^{\infty} \frac{\vec{P}_{n}(c)}{z^{n+1}},
$$

then it follows from (18) that

$$
\frac{1}{\lambda+\tau c} S_{\mu}\left(c, \frac{z}{\lambda+\tau c}\right)=\vec{S}_{P}(c, z) \cdot \vec{\mu}(c),
$$

or

$$
\vec{S}_{P}(c, z) \cdot \vec{\mu}(c)=\frac{1}{z}{ }_{p+1} F_{q+1}\left[\begin{array}{cc}
-\frac{z}{\lambda+\tau c}, & \vec{\alpha} \\
1-\frac{z}{\lambda+\tau c}, & \vec{\beta}+1
\end{array} ; c\right]
$$

\section{Examples}

In [15] we studied all families of semiclassical polynomials of class $s \leq 1$ orthogonal with respect to (1). When $s=0$, we have three canonical cases (the discrete classical polynomials):

\begin{tabular}{|c|c|c|}
\hline $\operatorname{deg}(\eta)$ & $\operatorname{deg}(\phi)$ & \\
\hline 0 & 1 & Charlier \\
\hline 1 & 1 & Meixner \\
\hline 2 & 2 & Hahn \\
\hline
\end{tabular}

where $\phi(x)$ and $\eta(x)$ were defined in (3). These polynomials are associated with the Poisson, Pascal, and hypergeometric probability distributions, and results about their moments have appeared in many places before (see [21], for instance). 
In [28], the authors used (11) and inversion formulas of the form

$$
(x-n+1)_{n}=\sum_{k=0}^{n} c_{n, k} Q_{k}(x),
$$

to derive expressions for the moments and exponential generating functions of the discrete classical polynomials. Stieltjes' transforms were not considered.

When $s=1$, we obtained five cases:

\begin{tabular}{|c|c|c|}
\hline $\operatorname{deg}(\eta)$ & $\operatorname{deg}(\phi)$ & \\
\hline 0 & 2 & generalized Charlier \\
\hline 1 & 2 & generalized Meixner \\
\hline 2 & 1 & generalized Krawtchouk \\
\hline 2 & 2 & generalized Hahn of type I \\
\hline 3 & 3 & generalized Hahn of type II \\
\hline
\end{tabular}

The moments of these polynomials have not (to our knowledge) been studied before.

\subsection{Charlier}

The Charlier polynomials [29, (18.20.8)]

$$
C_{n}(x ; c)={ }_{2} F_{0}\left(\begin{array}{c}
-n,-x \\
-
\end{array}-\frac{1}{c}\right)
$$

are orthogonal with respect to the weight function $[6]$

$$
\rho_{C}(x)=\frac{c^{x}}{x !}, \quad c>0 .
$$

In this case, we have

$$
\mu_{0}(c)={ }_{0} F_{0}[-; c]=e^{c},
$$

and hence

$$
\xi=0, \quad(\lambda, \tau)=(1,0) .
$$

From (13) we see that $\mu_{0}(c)$ satisfies the ODE

$$
(\vartheta-c) \mu_{0}=0,
$$

which implies $\mu_{1}=c \mu_{0}$. Thus, $\sigma_{0}(c)=c$, and Proposition 1 gives

$$
\mu_{n}(c)=P_{n}(c) \mu_{0}(c),
$$

with $P_{n}(c)$ defined by $P_{0}(c)=1$ and

$$
P_{n+1}=c \frac{d P_{n}}{d c}+c P_{n}
$$

The polynomials satisfying (25) are known as Bell (or Touchard, or exponential) polynomials. It is well known that they have the explicit representation [11]

$$
P_{n}(c)=\sum_{k=0}^{n}\left\{\begin{array}{l}
n \\
k
\end{array}\right\} c^{k},
$$


and therefore (see also [28, equation (44)])

$$
\mu_{n}(c)=e^{c} \sum_{k=0}^{n}\left\{\begin{array}{l}
n \\
k
\end{array}\right\} c^{k},
$$

in agreement with (10).

Using (19) and (20), we see that the generating functions of $\mu_{n}(c)$ and $P_{n}(c)$ are given by (see also [28, equation (50)])

$$
G_{\mu}(c, w)=\mu_{0}\left(c e^{w}\right)=e^{c e^{w}}
$$

and

$$
G_{P}(c, w)=\frac{e^{c e^{w}}}{e^{c}}=e^{c\left(e^{w}-1\right)} .
$$

\subsection{Meixner}

The Meixner polynomials [29, (18.20.7)]

$$
M_{n}(x ; \alpha, c)={ }_{2} F_{1}\left(\begin{array}{c}
-n,-x \\
\alpha
\end{array} 1-\frac{1}{c}\right)
$$

are orthogonal with respect to the weight function $[29,(18.19)]$

$$
\rho_{M}(x ; \alpha, c)=(\alpha)_{x} \frac{c^{x}}{x !}, \quad 0<c<1, \quad \alpha>0 .
$$

In this case, we have

$$
\mu_{0}^{M}(c ; \alpha)={ }_{1} F_{0}\left[\begin{array}{l}
\alpha \\
-
\end{array} c\right]=(1-c)^{-\alpha},
$$

and hence

$$
\xi=0, \quad(\lambda, \tau)=(1,-1) .
$$

From (13) we see that $\mu_{0}^{M}(c ; \alpha)$ satisfies the ODE

$$
[\vartheta-c(\vartheta+\alpha)] \mu_{0}^{M}=0,
$$

which implies

$$
(1-c) \mu_{1}^{M}=\alpha c \mu_{0}^{M} .
$$

Thus, $\sigma_{0}(c)=\alpha c$, and Proposition 1 gives

$$
\mu_{n}^{M}(c ; \alpha)=(1-c)^{-n} P_{n}^{M}(c) \mu_{0}^{M}(c ; \alpha),
$$

with $P_{n}^{M}(c)$ defined by $P_{0}^{M}(c)=1$ and (see also [27, equation (5.68)])

$$
P_{n+1}^{M}=c(1-c) \frac{d P_{n}^{M}}{d c}+(\alpha+n) c P_{n}^{M} .
$$

From (10), we have (see also [28, equation (43)])

$$
\mu_{n}^{M}(c ; \alpha)=\sum_{k=0}^{n}\left\{\begin{array}{l}
n \\
k
\end{array}\right\} c^{k}(\alpha)_{k}(1-c)^{-(\alpha+k)},
$$


and therefore

$$
P_{n}^{M}(c)=\sum_{k=0}^{n}\left\{\begin{array}{l}
n \\
k
\end{array}\right\}(\alpha)_{k} c^{k}(1-c)^{n-k} .
$$

Using (19) and (20), we see that the generating functions of $\mu_{n}^{M}(c ; \alpha)$ and $P_{n}^{M}(c)$ are given by (see also [28, equation (49)])

$$
G_{\mu}(c, w)=\mu_{0}\left(c e^{w}\right)=\left(1-c e^{w}\right)^{-\alpha},
$$

and

$$
G_{P}(c, w)=\frac{\left[1-c e^{(1-c) w}\right]^{-\alpha}}{(1-c)^{-\alpha}}=\left[\frac{1-c e^{(1-c) w}}{1-c}\right]^{-\alpha} .
$$

\subsubsection{Krawtchouk polynomials}

The Krawtchouk polynomials [29, (18.20.6)]

$$
K_{n}(x ; \mathfrak{p}, N)={ }_{2} F_{1}\left(\begin{array}{c}
-n,-x \\
-N
\end{array} ; \frac{1}{\mathfrak{p}}\right),
$$

are orthogonal with respect to the weight function [9]

$$
\rho_{K}(x ; \mathfrak{p}, N)=\left(\begin{array}{c}
N \\
x
\end{array}\right) \mathfrak{p}^{x}(1-\mathfrak{p})^{N-x}, \quad N \in \mathbb{N}, \quad 0<\mathfrak{p}<1 .
$$

It is related to the weight function of the Meixner polynomials (27) by

$$
\rho_{K}(x ; \mathfrak{p}, N)=(1-\mathfrak{p})^{N} \frac{(-N)_{x}}{x !}\left(\frac{\mathfrak{p}}{\mathfrak{p}-1}\right)^{x}=(1-\mathfrak{p})^{N} \rho_{M}\left(x ;-N, \frac{\mathfrak{p}}{\mathfrak{p}-1}\right) .
$$

Using (32) in (28), we obtain

$$
\mu_{0}^{K}(\mathfrak{p})=(1-\mathfrak{p})^{N} \mu_{0}^{M}\left(-N, \frac{\mathfrak{p}}{\mathfrak{p}-1}\right)=1 .
$$

and using (29) and (31), we get $\mu_{n}^{K}(\mathfrak{p})=P_{n}^{K}(\mathfrak{p})$, with (see also [28, equation (42)])

$$
P_{n}^{K}(\mathfrak{p})=\sum_{k=0}^{n}\left\{\begin{array}{l}
n \\
k
\end{array}\right\}\left(\begin{array}{l}
N \\
k
\end{array}\right) k ! \mathfrak{p}^{k} .
$$

From (30) and (32), we have

$$
P_{n+1}^{K}=\mathfrak{p}(1-\mathfrak{p}) \frac{d P_{n}^{K}}{d \mathfrak{p}}+N \mathfrak{p} P_{n}^{K} .
$$

To obtain the generating function of $P_{n}^{K}(\mathfrak{p})$, we can use [29, (26.8.12)]

$$
\sum_{n=0}^{\infty}\left\{\begin{array}{l}
n \\
k
\end{array}\right\} \frac{w^{n}}{n !}=\frac{\left(e^{w}-1\right)^{k}}{k !}
$$

and we get (see also $[28$, equation (48)])

$$
G_{P}(\mathfrak{p}, w)=\left[1+\left(e^{w}-1\right) \mathfrak{p}\right]^{N} .
$$




\subsection{Generalized Charlier}

These polynomials are orthogonal with respect to the weight function [17]

$$
\rho(x ; \beta, c)=\frac{1}{(\beta+1)_{x}} \frac{c^{x}}{x !}, \quad c>0, \quad \beta>-1 .
$$

In this case, we have

$$
\mu_{0}(c)={ }_{0} F_{1}[\beta+1 ; c]=c^{-\frac{\beta}{2}} \Gamma(\beta+1) I_{\beta}(2 \sqrt{c}),
$$

where $I_{\beta}(z)$ is the modified Bessel function of the first kind [29, (10.39.9)], and hence

$$
\xi=1, \quad(\lambda, \tau)=(1,0) .
$$

From (13) we see that $\mu_{0}(c)$ satisfies the ODE

$$
\vartheta(\vartheta+\beta) \mu_{0}-c \mu_{0}=0,
$$

which implies

$$
\mu_{2}=c \mu_{0}-\beta \mu_{1},
$$

and therefore

$$
\sigma_{0}(c)=c, \quad \sigma_{1}(c)=-\beta .
$$

Proposition 1 gives

$$
\mu_{n}(c)=\vec{P}_{n}(c) \cdot \vec{\mu}(c),
$$

with $\vec{P}_{n}(c)$ defined by (16) and

$$
\vec{P}_{n+1}=c \frac{d \vec{P}_{n}}{d c}+\left[\begin{array}{cc}
0 & c \\
1 & -\beta
\end{array}\right] \vec{P}_{n} .
$$

To obtain the Stieltjes transform of $\vec{P}_{n}(c)$, let's write

$$
\vec{P}_{n}(c)=\left[\begin{array}{l}
Q_{n}(c) \\
R_{n}(c)
\end{array}\right], \quad \vec{S}_{P}(c, z)=\left[\begin{array}{l}
U(c, z) \\
V(c, z)
\end{array}\right] .
$$

From (34), we have

$$
Q_{n+1}=c Q_{n}^{\prime}+c R_{n}, \quad R_{n+1}=c R_{n}^{\prime}-\beta R_{n}+Q_{n},
$$

with $Q_{0}=1, R_{0}=0$. Using (21), we get

$$
z U-1=c \frac{\partial U}{\partial c}+c V, \quad z V=c \frac{\partial V}{\partial c}-\beta V+U .
$$

The solution of the system (35) is given by

$$
U(c, z)=\frac{1}{z}{ }_{1} F_{2}\left[\begin{array}{c}
1 \\
1-z,-\beta-z
\end{array} ; c\right], \quad V(c, z)=\frac{1}{z(z+\beta)}{ }_{1} F_{2}\left[\begin{array}{c}
1 \\
1-z, 1-\beta-z
\end{array} ; c\right] .
$$

In this case

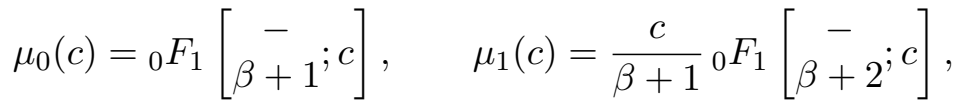

and since

$$
\begin{aligned}
& \frac{1}{z}{ }_{1} F_{2}\left[\begin{array}{c}
1 \\
1-z,-\beta-z
\end{array} ; c\right]{ }_{0} F_{1}\left[\begin{array}{c}
- \\
\beta+1
\end{array} ; c\right] \\
& +\frac{1}{z(z+\beta)}{ }_{1} F_{2}\left[\begin{array}{c}
1 \\
1-z, 1-\beta-z
\end{array} ; c\right] \frac{c}{\beta+1}{ }_{0} F_{1}\left[\begin{array}{c}
- \\
\beta+2
\end{array} ; c\right]=\frac{1}{z}{ }_{1} F_{2}\left[\begin{array}{c}
-z \\
1-z, \beta+1
\end{array} ; c\right],
\end{aligned}
$$

we see that (24) is satisfied. 


\subsection{Generalized Meixner}

These polynomials are orthogonal with respect to the weight function [3]

$$
\rho(x ; \alpha, \beta, c)=\frac{(\alpha)_{x}}{(\beta+1)_{x}} \frac{c^{x}}{x !}, \quad c, \alpha>0, \quad \beta>-1 .
$$

In this case, we have

$$
\mu_{0}(c)={ }_{1} F_{1}\left[\begin{array}{c}
\alpha \\
\beta+1
\end{array} ; c\right]=M(\alpha, \beta+1, c)
$$

where $M(a, b, c)$ is Kummer's (confluent hypergeometric) function [29, (13.2.2)], and hence

$$
\xi=1, \quad(\lambda, \tau)=(1,0) .
$$

From (13) we see that $\mu_{0}(c)$ satisfies the ODE

$$
\vartheta(\vartheta+\beta) \mu_{0}-c(\vartheta+\alpha) \mu_{0}=0,
$$

which implies

$$
\mu_{2}=c \alpha \mu_{0}+(c-\beta) \mu_{1},
$$

and therefore

$$
\sigma_{0}(c)=\alpha c, \quad \sigma_{1}(c)=c-\beta .
$$

Proposition 1 gives

$$
\mu_{n}(c)=\vec{P}_{n}(c) \cdot \vec{\mu}(c),
$$

with $\vec{P}_{n}(c)$ defined by (16) and

$$
\vec{P}_{n+1}=c \frac{d \vec{P}_{n}}{d c}+\left[\begin{array}{cc}
0 & \alpha c \\
1 & c-\beta
\end{array}\right] \vec{P}_{n}
$$

To obtain the Stieltjes transform of $\vec{P}_{n}(c)$, let's write

$$
\vec{P}_{n}(c)=\left[\begin{array}{l}
Q_{n}(c) \\
R_{n}(c)
\end{array}\right], \quad \vec{S}_{P}(c, z)=\left[\begin{array}{l}
U(c, z) \\
V(c, z)
\end{array}\right] .
$$

From (34), we have

$$
Q_{n+1}=c Q_{n}^{\prime}+\alpha c R_{n}, \quad R_{n+1}=c R_{n}^{\prime}+(c-\beta) R_{n}+Q_{n},
$$

with $Q_{0}=1, R_{0}=0$. Using (21), we get

$$
z U-1=c \frac{\partial U}{\partial c}+\alpha c V, \quad z V=c \frac{\partial V}{\partial c}+(c-\beta) V+U .
$$

If we represent the functions $U, V$ as

$$
U(c, z)=\sum_{n=0}^{\infty} u_{n}(z) c^{n}, \quad V(c, z)=\sum_{n=0}^{\infty} v_{n}(z) c^{n}
$$


then (36) gives

$$
z u_{n}-\delta_{n, 0}=n u_{n}+\alpha v_{n-1}, \quad z v_{n}=n v_{n}+v_{n-1}-\beta v_{n}+u_{n} .
$$

We have (assuming that $u_{n}=v_{n}=0$ for $n<0$ )

$$
u_{0}=\frac{1}{z}, \quad v_{0}=\frac{1}{z(z+\beta)},
$$

and

$$
u_{n}=(z+\beta-n) v_{n}-v_{n-1} .
$$

Using (38) in (37), we obtain

$$
v_{n}=\frac{z+\alpha-n}{(z-n)(z+\beta-n)} v_{n-1}, \quad n=1,2, \ldots,
$$

and therefore

$$
v_{n}=\frac{(-1)^{n}}{z(z+\beta)} \frac{(1-\alpha-z)_{n}}{(1-z)_{n}(1-\beta-z)_{n}}, \quad n=0,1, \ldots
$$

From (38), it follows that

$$
u_{n}=\alpha \frac{(-1)^{n}}{z(z+\alpha)} \frac{(-\alpha-z)_{n}}{(1-z)_{n}(-\beta-z)_{n}}, \quad n=1,2, \ldots
$$

Thus,

$$
\begin{aligned}
U(c, z) & =\frac{1}{z}+\frac{\alpha}{z(z+\alpha)} \sum_{n=1}^{\infty} \frac{(-\alpha-z)_{n}}{(1-z)_{n}(-\beta-z)_{n}}(-c)^{n} \\
& =\frac{1}{z}-\frac{\alpha}{z(z+\alpha)}+\frac{\alpha}{z(z+\alpha)} \sum_{n=0}^{\infty} \frac{(-\alpha-z)_{n}}{(1-z)_{n}(-\beta-z)_{n}}(-c)^{n} \\
& =\frac{1}{z+\alpha}+\frac{\alpha}{z(z+\alpha)}{ }_{2} F_{2}\left[\begin{array}{c}
1,-\alpha-z \\
1-z,-\beta-z
\end{array} ;-c\right]
\end{aligned}
$$

and

$$
V(c, z)=\frac{1}{z(z+\beta)}{ }_{2} F_{2}\left[\begin{array}{c}
1,1-\alpha-z \\
1-z, 1-\beta-z
\end{array} ;-c\right] .
$$

In this case

$$
\mu_{0}(c)={ }_{1} F_{1}\left[\begin{array}{c}
\alpha \\
\beta+1
\end{array} ; c\right], \quad \mu_{1}(c)=\frac{\alpha c}{\beta+1}{ }_{1} F_{1}\left[\begin{array}{l}
\alpha+1 \\
\beta+2
\end{array} ; c\right],
$$

and since

$$
\begin{aligned}
& \left(\frac{1}{z+\alpha}+\frac{\alpha}{z(z+\alpha)}{ }_{2} F_{2}\left[\begin{array}{c}
1,-\alpha-z \\
1-z,-\beta-z
\end{array} ;-c\right]\right){ }_{1} F_{1}\left[\begin{array}{c}
\alpha \\
\beta+1
\end{array} ; c\right] \\
& \quad+\frac{1}{z(z+\beta)}{ }_{2} F_{2}\left[\begin{array}{c}
1,1-\alpha-z \\
1-z, 1-\beta-z
\end{array} ;-c\right] \frac{\alpha c}{\beta+1}{ }_{1} F_{1}\left[\begin{array}{l}
\alpha+1 \\
\beta+2
\end{array} ; c\right]=\frac{1}{z}{ }_{2} F_{2}\left[\begin{array}{c}
-z, \alpha \\
1-z, \beta+1
\end{array} ; c\right],
\end{aligned}
$$

we see that (24) is satisfied. 


\subsection{Generalized Krawtchouk}

These polynomials are orthogonal with respect to the weight function [15]

$$
\rho(x ; \alpha, N, c)=(\alpha)_{x}(-N)_{x} \frac{c^{x}}{x !}, \quad c<0, \quad \alpha>0, \quad N \in \mathbb{N} .
$$

In this case, we have

$$
\mu_{0}(c)={ }_{2} F_{0}\left[\begin{array}{c}
\alpha,-N \\
-
\end{array} ; c\right]=C_{N}\left(-\alpha ;-c^{-1}\right)
$$

where $C_{N}(x ; \mu)$ is the Charlier polynomial [6], and hence

$$
\xi=1, \quad(\lambda, \tau)=(0,1) .
$$

From (13) we see that $\mu_{0}(c)$ satisfies the ODE

$$
\vartheta \mu_{0}-c(\vartheta+\alpha)(\vartheta-N) \mu_{0}=0,
$$

which implies

$$
c \mu_{2}=\alpha N c \mu_{0}+(N c-\alpha c+1) \mu_{1},
$$

and therefore

$$
\sigma_{0}(c)=\alpha N c, \quad \sigma_{1}(c)=(N-\alpha) c+1 .
$$

Proposition 1 gives

$$
\mu_{n}(c)=c^{-n} \vec{P}_{n}(c) \cdot \vec{\mu}(c),
$$

with $\vec{P}_{n}(c)$ defined by (16) and

$$
\vec{P}_{n+1}=c^{2} \frac{d \vec{P}_{n}}{d c}+\left[\begin{array}{cc}
-n c & \alpha N c \\
c & (N-\alpha-n) c+1
\end{array}\right] \vec{P}_{n}(c) .
$$

\subsection{Generalized Hahn polynomials of type I}

These polynomials are orthogonal with respect to the weight function [15]

$$
\rho\left(x ; \alpha_{1}, \alpha_{2}, \beta ; c\right)=\frac{\left(\alpha_{1}\right)_{x}\left(\alpha_{2}\right)_{x}}{(\beta+1)_{x}} \frac{c^{x}}{x !},
$$

where $0<c<1, \alpha_{1}, \alpha_{2}>0, \beta>-1$. In this case, we have

$$
\mu_{0}(c)={ }_{2} F_{1}\left[\begin{array}{c}
\alpha_{1}, \alpha_{2} \\
\beta+1
\end{array} ;\right],
$$

and hence

$$
\xi=1, \quad(\lambda, \tau)=(1,-1) .
$$

From (13) we see that $\mu_{0}(c)$ satisfies the ODE

$$
\vartheta(\vartheta+\beta) \mu_{0}-c\left(\vartheta+\alpha_{1}\right)\left(\vartheta+\alpha_{2}\right) \mu_{0}=0,
$$


which implies

$$
(1-c) \mu_{2}=\alpha_{1} \alpha_{2} c \mu_{0}+\left[\left(\alpha_{1}+\alpha_{2}\right) c-\beta\right] \mu_{1},
$$

and therefore

$$
\sigma_{0}(c)=\alpha_{1} \alpha_{2} c, \quad \sigma_{1}(c)=\left(\alpha_{1}+\alpha_{2}\right) c-\beta .
$$

Proposition 1 gives

$$
\mu_{n}(c)=(1-c)^{-n} \vec{P}_{n}(c) \cdot \vec{\mu}(c),
$$

with $\vec{P}_{n}(c)$ defined by (16) and

$$
\vec{P}_{n+1}=c(1-c) \frac{d \vec{P}_{n}}{d c}+\left[\begin{array}{cc}
n c & \alpha_{1} \alpha_{2}(1-c) c \\
1-c & \left(\alpha_{1}+\alpha_{2}+n\right) c-\beta
\end{array}\right] \vec{P}_{n}
$$

\subsection{Hahn polynomials}

The Hahn polynomials [29, (18.20.5)]

$$
H_{n}(x ; \alpha, \beta, N)={ }_{3} F_{2}\left(\begin{array}{c}
-n,-x, n+\alpha+\beta+1 \\
-N, \alpha+1
\end{array} ; 1\right)
$$

are orthogonal with respect to the weight function [29, (18.19)]

$$
\rho_{H}(x ; \alpha, \beta, N)=\frac{(\alpha+1)_{x}}{x !} \frac{(\beta+1)_{N-x}}{(N-x) !}, \quad \alpha, \beta \notin[-N,-1], \quad N \in \mathbb{N},
$$

or

$$
\rho_{H}(x ; \alpha, \beta, N)=\left(\begin{array}{c}
\alpha+x \\
x
\end{array}\right)\left(\begin{array}{c}
\beta+N-x \\
N-x
\end{array}\right) .
$$

The relation between $\rho_{H}(x ; \alpha, \beta, N)$ and the weight function of the generalized Hahn polynomials (39) is given by

$$
\rho_{H}(x ; \alpha, \beta, N)=\frac{(\beta+1)_{N}}{N !} \rho(x ; \alpha+1,-N,-N-\beta-1 ; 1) .
$$

From (10), we get (see also [28, equation (41)])

$$
\mu_{n}^{H}=\sum_{k=0}^{n}\left\{\begin{array}{l}
n \\
k
\end{array}\right\}(\alpha+1)_{k} \frac{(\alpha+\beta+2+k)_{N-k}}{(N-k) !},
$$

where we have used

$$
\mu_{0}^{H}=\frac{(\alpha+\beta+2)_{N}}{N !} .
$$

To obtain the generating function of $\mu_{n}^{H}$, we can use (33), and we get

$$
G_{\mu^{H}}(z)=\frac{(\alpha+\beta+2)_{N}}{N !}{ }_{2} F_{1}\left[\begin{array}{c}
-N, \alpha+1 \\
\alpha+\beta+2
\end{array} ; 1-e^{z}\right] .
$$

This generating function seems not to have been considered before.

Remark 1. Except for the Bell polynomials (26), all the other families $\vec{P}_{n}(c)$ associated to the moments $\mu_{n}(c)$ seem to be new.

Remark 2. Stieltjes transforms of the generalized Krawtchouk and generalized Hahn polynomials of type I have been omitted because of the complexity of the formulas. 


\section{Conclusion}

We have developed a technique for computing the moments of weight functions of hypergeometric type. We have shown that the moments are linear combinations of the first $\xi+1$ moments with polynomial coefficients in the parameter $c$. We have also constructed generating functions for both the moments and the polynomials associated with them.

All the results in Sections 3.3-3.5 are new, and give efficient ways of computing the moments of the discrete semiclassical polynomials of class 1 . The same method can be used to find the moments of polynomials of class $s>1$.

In a previous work [16], we found the asymptotic zero distribution of polynomial families satisfying first-order differential-recurrence relations of the form (12). It would be interesting to know if our results could be extended to include the polynomials $\vec{P}_{n}(c)$ studied in this paper.

\section{Acknowledgements}

We want to thank the anonymous referees and the editors of this special issue for providing us with extremely valuable suggestions that helped us to greatly improve our first draft of the paper.

\section{References}

[1] Akhiezer N.I., The classical moment problem and some related questions in analysis, Hafner Publishing Co., New York, 1965.

[2] Andrews G.E., Askey R., Roy R., Special functions, Encyclopedia of Mathematics and its Applications, Vol. 71, Cambridge University Press, Cambridge, 1999.

[3] Boelen L., Filipuk G., Van Assche W., Recurrence coefficients of generalized Meixner polynomials and Painlevé equations, J. Phys. A: Math. Theor. 44 (2011), 035202, 19 pages.

[4] Chihara T.S., An introduction to orthogonal polynomials, Mathematics and its Applications, Vol. 13, Gordon and Breach Science Publishers, New York - London - Paris, 1978.

[5] Clarkson P.A., Recurrence coefficients for discrete orthonormal polynomials and the Painlevé equations, J. Phys. A: Math. Theor. 46 (2013), 185205, 18 pages, arXiv:1301.2396.

[6] Dominici D., Asymptotic analysis of the Askey-scheme. I. From Krawtchouk to Charlier, Cent. Eur. J. Math. 5 (2007), 280-304, math.CA/0501072.

[7] Dominici D., Asymptotic analysis of the Hermite polynomials from their differential-difference equation, J. Difference Equ. Appl. 13 (2007), 1115-1128, math.CA/0601078.

[8] Dominici D., Asymptotic analysis of generalized Hermite polynomials, Analysis (Munich) 28 (2008), 239261, math.CA/0606324.

[9] Dominici D., Asymptotic analysis of the Krawtchouk polynomials by the WKB method, Ramanujan J. 15 (2008), 303-338, math.CA/0501042.

[10] Dominici D., Some properties of the inverse error function, in Tapas in Experimental Mathematics, Contemp. Math., Vol. 457, Amer. Math. Soc., Providence, RI, 2008, 191-203.

[11] Dominici D., Asymptotic analysis of the Bell polynomials by the ray method, J. Comput. Appl. Math. 233 (2009), 708-718, arXiv:0709.0252.

[12] Dominici D., Mehler-Heine type formulas for Charlier and Meixner polynomials, Ramanujan J. 39 (2016), 271-289, arXiv:1406.6193.

[13] Dominici D., Driver K., Jordaan K., Polynomial solutions of differential-difference equations, J. Approx. Theory 163 (2011), 41-48, arXiv:0902.0041.

[14] Dominici D., Knessl C., Asymptotic analysis of a family of polynomials associated with the inverse error function, Rocky Mountain J. Math. 42 (2012), 847-872, arXiv:0811.2243.

[15] Dominici D., Marcellán F., Discrete semiclassical orthogonal polynomials of class one, Pacific J. Math. 268 (2014), 389-411, arXiv:1211.2005. 
[16] Dominici D., Van Assche W., Zero distribution of polynomials satisfying a differential-difference equation, Anal. Appl. (Singap.) 12 (2014), 635-666, arXiv:1312.0698.

[17] Filipuk G., Van Assche W., Recurrence coefficients of generalized Charlier polynomials and the fifth Painlevé equation, Proc. Amer. Math. Soc. 141 (2013), 551-562, arXiv:1106.2959.

[18] Hildebrandt E.H., Systems of polynomials connected with the Charlier expansions and the Pearson differential and difference equations, Ph.D. Thesis, University of Michigan, 1932.

[19] Ismail M.E.H., Stanton D., Classical orthogonal polynomials as moments, Canad. J. Math. 49 (1997), $520-542$.

[20] Ismail M.E.H., Stanton D., More orthogonal polynomials as moments, in Mathematical Essays in Honor of Gian-Carlo Rota (Cambridge, MA, 1996), Progr. Math., Vol. 161, Birkhäuser Boston, Boston, MA, 1998, 377-396.

[21] Johnson N.L., Kotz S., Kemp A.W., Univariate discrete distributions, 2nd ed., Wiley Series in Probability and Mathematical Statistics: Applied Probability and Statistics, John Wiley \& Sons, Inc., New York, 1992.

[22] Kemp A.W., Studies in univariate discrete distribution theory based on the generalized hypergeometric function and associated differential equations, Ph.D. Thesis, The Queen's University of Belfast, 1968.

[23] Koekoek R., Lesky P.A., Swarttouw R.F., Hypergeometric orthogonal polynomials and their $q$-analogues, Springer Monographs in Mathematics, Springer-Verlag, Berlin, 2010.

[24] Kreı̆n M.G., Nudel'man A.A., The Markov moment problem and extremal problems, Translations of Mathematical Monographs, Vol. 50, Amer. Math. Soc., Providence, R.I., 1977.

[25] Maroni P., Une théorie algébrique des polynômes orthogonaux. Application aux polynômes orthogonaux semi-classiques, in Orthogonal Polynomials and their Applications (Erice, 1990), IMACS Ann. Comput. Appl. Math., Vol. 9, Baltzer, Basel, 1991, 95-130.

[26] Mohammad-Noori M., Some remarks about the derivation operator and generalized Stirling numbers, Ars Combin. 100 (2011), 177-192, arXiv:1012.3948.

[27] Njionou Sadjang P., Moments of classical orthogonal polynomials, Ph.D. Thesis, Universität Kassel, 2013.

[28] Njionou Sadjang P., Koepf W., Foupouagnigni M., On moments of classical orthogonal polynomials, J. Math. Anal. Appl. 424 (2015), 122-151.

[29] Olver F.W.J., Lozier D.W., Boisvert R.F., Clark C.W. (Editors), NIST handbook of mathematical functions, U.S. Department of Commerce, National Institute of Standards and Technology, Washington, DC, Cambridge University Press, Cambridge, 2010.

[30] Pearson K., Contributions to the mathematical theory of evolution. II. Skew variation in homogeneous material, Philos. Trans. Roy. Soc. London Ser. A 186 (1895), 343-414.

[31] Schoutens W., Stochastic processes and orthogonal polynomials, Lecture Notes in Statistics, Vol. 146, Springer-Verlag, New York, 2000.

[32] Shohat J.A., Tamarkin J.D., The problem of moments, American Mathematical Society Mathematical Surveys, Vol. 1, Amer. Math. Soc., New York, 1943.

[33] Wilf H.S., Generatingfunctionology, 3rd ed., A K Peters, Ltd., Wellesley, MA, 2006. 